\title{
An obstructing rectal gastrointestinal stromal tumour diagnosed using endoscopic ultrasonography
}

Paul J Belletrutti MD FRCPC, Lorne Price MD FRCPC

\section{CASE PRESENTATION}

A 53-year-old Caucasian man presented with a several-month history of progressive obstipation. At the time of presentation to hospital, he had been unable to have a bowel movement for 10 days. He was otherwise healthy with no other medical diagnoses. He was not taking any medications but was an active cigarette smoker.

On digital rectal examination, a large, smooth, rounded mass was immediately palpable in the rectal vault. At proctoscopy, a large mass with edematous overlying mucosa occupied the rectum. Mucosal biopsies showed reactive inflammatory changes but no evidence of neoplasia. A computed tomography scan delineated a solid $9 \mathrm{~cm} \times 7 \mathrm{~cm}$ mass in the pelvis suspected to be of prostatic or urothelial origin (Figure 1). However, rectal endoscopic ultrasonography (EUS) depicted a hypoechoic mass clearly arising from muscularis propria layer of the rectal wall (Figure 2, red arrow) with a clear plane apparent between the mass and prostate gland (Figure 2, green arrow).

EUS-guided fine-needle aspiration cytology revealed a spindle cell neoplasm that stained positively for CD-117 (c-KIT) and CD-34, confirming the diagnosis of a gastrointestinal (GI) stromal tumour (GIST). The patient received neodjuvant imatinib therapy with a resultant $50 \%$ reduction in tumour volume. The hope was to perform a sphincter preserving resection; however, a complete mesorectal and perineal resection was required due to tumour adherence. On final pathology, a $4.6 \mathrm{~cm} \times 5 \mathrm{~cm}$ GIST was confirmed (Figure 3). The mitotic count was $1 / 50$ high-powered field; thus, adjuvant imatinib was deferred. Further tumour testing revealed an exon-11 KIT gene mutation. The patient is currently alive and functioning well 12 months later.

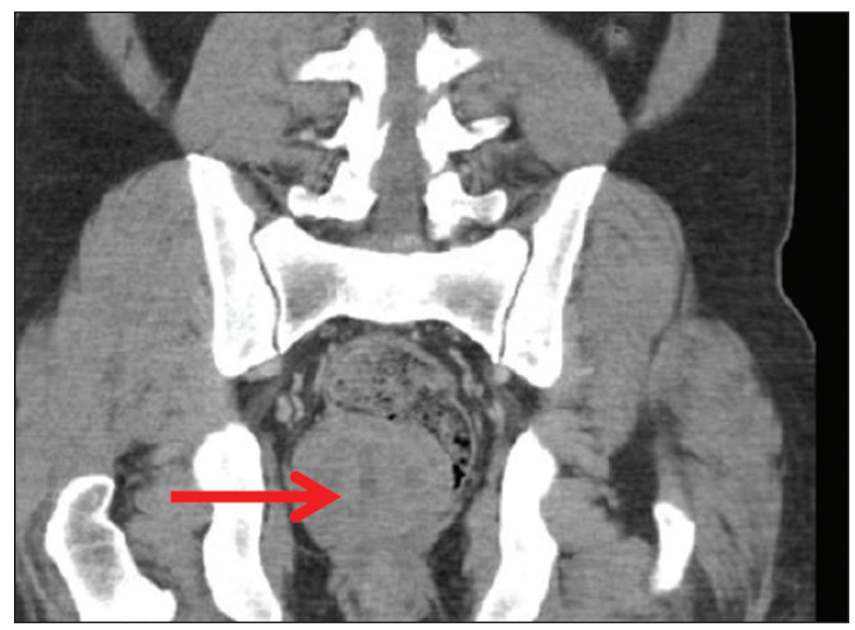

Figure 1) Coronal computed tomography image revealing a large solid tumour (arrow) obstructing the rectum

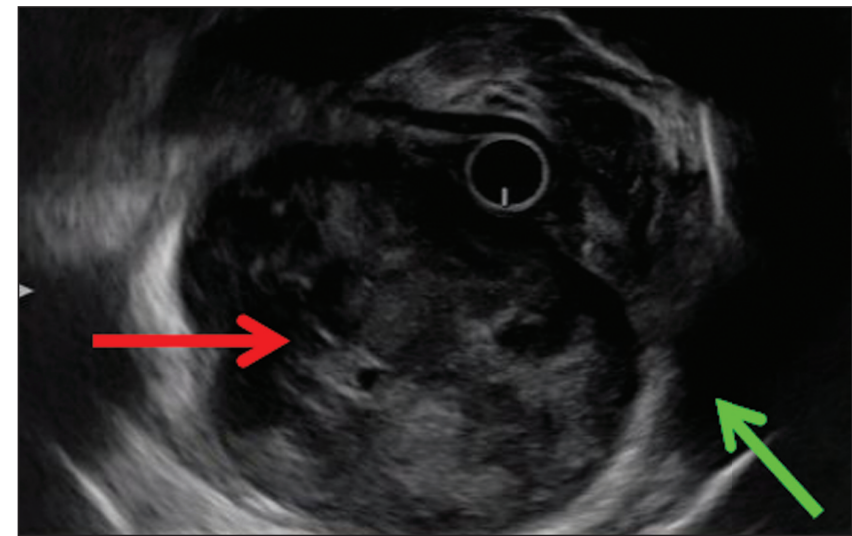

Figure 2) Radial endoscopic ultrasonography view showing a well-demarcated hypoechoic mass (red arrow) arising from the rectal wall with a clear plane between the mass and prostate gland (green arrow)

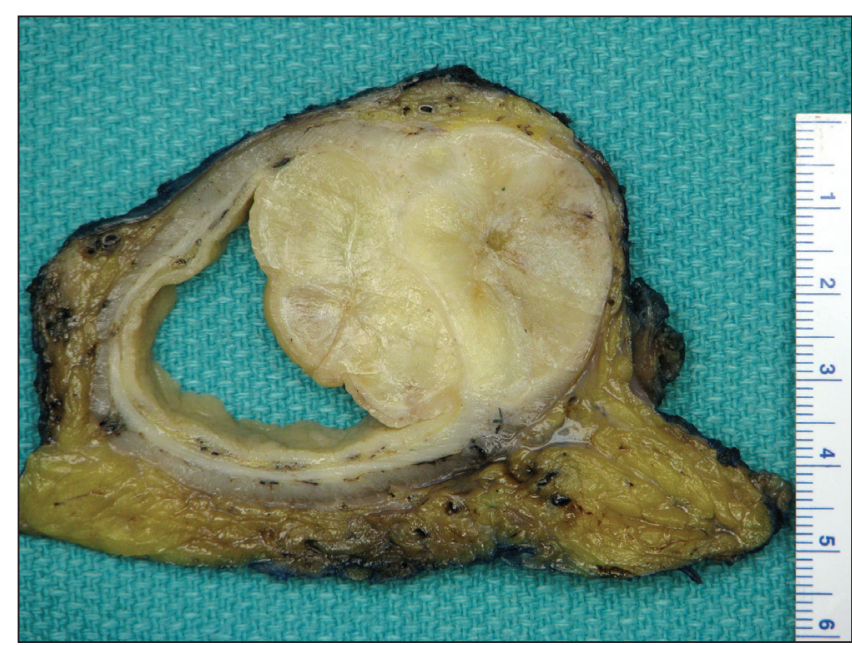

Figure 3) Cross-section through resected specimen showing large rectal wall gastrointestinal tumour

\section{DISCUSSION}

Although GISTs are the most common mesenchymal tumour of the GI tract, only $5 \%$ arise from the colorectum (1). Approximately $70 \%$ of GISTs are associated with clinical symptoms, namely GI bleeding, obstruction or abdominal pain (2). Eighty-five percent of GISTs harbour activating mutations in the KIT transmembrane receptor, usually in exon 11; this serves as the molecular target for tyrosine kinase inhibitors

Foothills Medical Center, Division of Gastroenterology and Hepatology, Department of Medicine, University of Calgary, Calgary, Alberta

Correspondence: Dr Paul J Belletrutti, Division of Gastroenterology and Hepatology, University of Calgary, Teaching, Research and Wellness Building-6D23,

3280 Hospital Drive Northwest, Calgary, Alberta T2N 4Z6. Telephone 403-592-5089, fax 403-592-5090, e-mail pjbellet@ucalgary.ca

Received for publication February 13, 2015. Accepted February 27, 2015 
such as imatinib and sunitinib (3). Our case highlights typical GIST features arising from an unusual location. EUS was the key investigation to make the diagnosis preoperatively and provide the opportunity for neoadjuvant therapy. EUS can accurately delineate the origin of pelvic tumours around the rectum and enables safe tissue acquisition. Cell blocks from cytology specimens can be further characterized by immunohistochemistry. Therefore, although rare, a rectal GIST should be considered in a patient presenting with rectal outlet obstruction.

DISCLOSURES: The authors have no financial disclosures or conflicts of interest to declare.

\section{REFERENCES}

1. Liegl-Atzwanger B, Fletcher JA, Fletcher CD. Gastrointestinal stromal tumors. Virchows Arch 2010;456:111-27.

2. Nilsson B, Bumming P, Meis-Kindblom JM, et al. Gastrointestinal stromal tumors: The incidence, prevalence, clinical course, and prognostication in the preimatinib mesylate era - a populationbased study in western Sweden. Cancer 2005;103:821-9.

3. Corless CL, Fletcher JA, Heinrich MC. Biology of gastrointestinal stromal tumors. J Clin Oncol 2004;22:3813-25.

The Canadian Journal of Gastroenterology $\mathcal{E}$ Hepatology is considering a limited number of submissions for IMAGE OF THE MONTH. These are based on endoscopic, histological, radiological and/or patient images, which must be anonymous with no identifying features visible. The patient must consent to publication and the consent must be submitted with the manuscript. All manuscripts should be practical and relevant to clinical practice, and not simply a case report of an esoteric condition. The text should be brief, structured as CASE PRESENTATION and DISCUSSION, and not more than 700 words in length. A maximum of three images can be submitted and the number of references should not exceed five. The submission may be edited by our editorial team. 


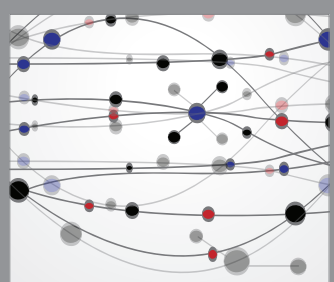

The Scientific World Journal
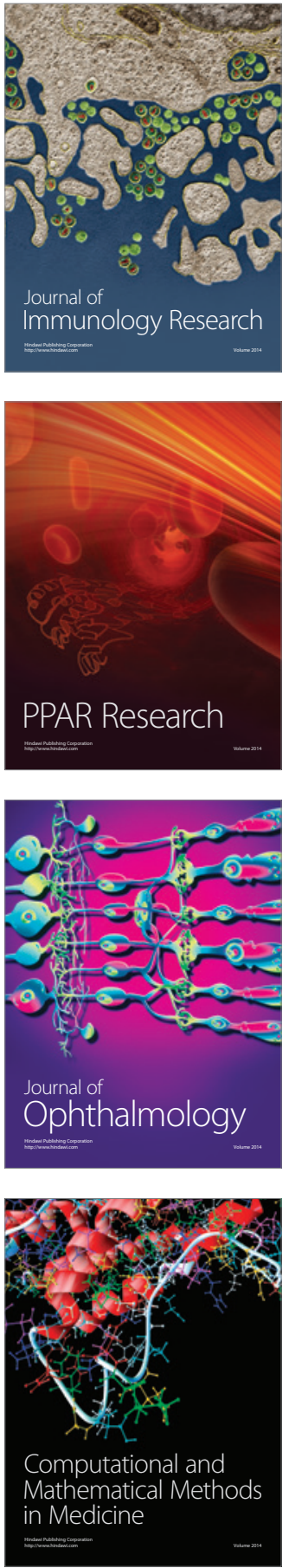

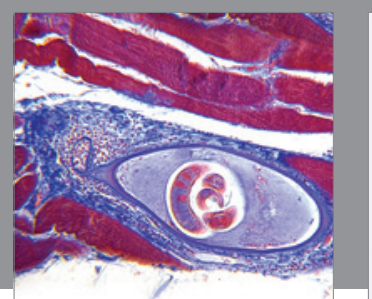

Gastroenterology Research and Practice

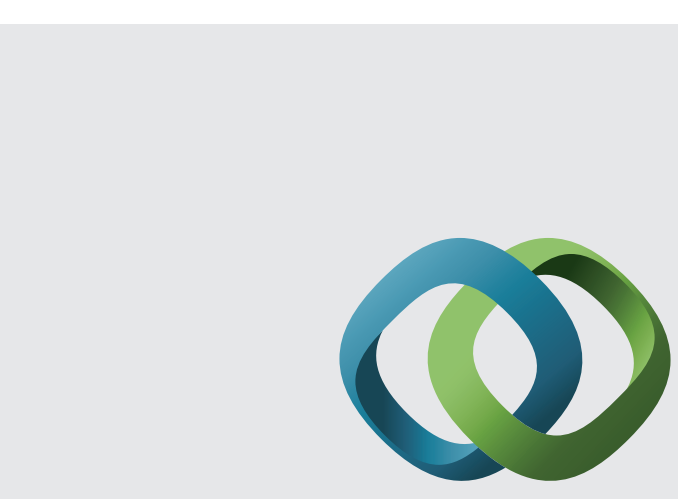

\section{Hindawi}

Submit your manuscripts at

http://www.hindawi.com
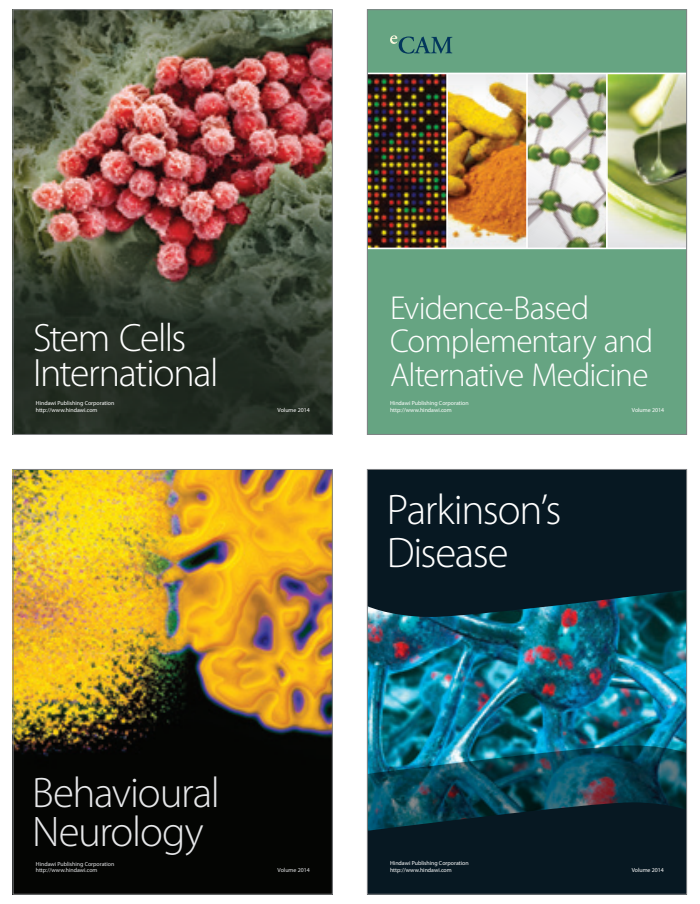
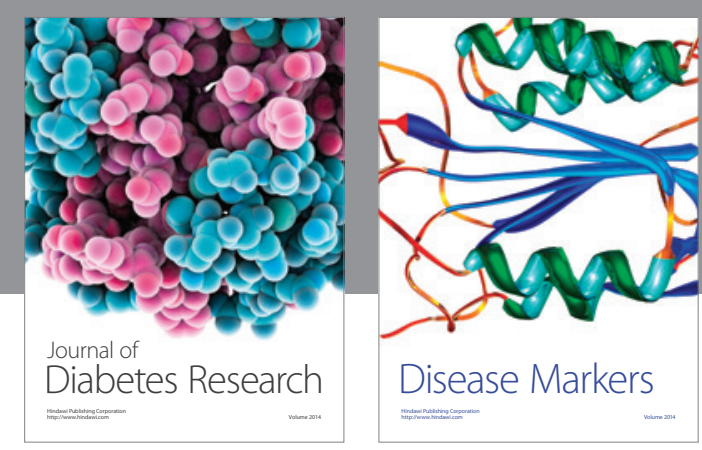

Disease Markers
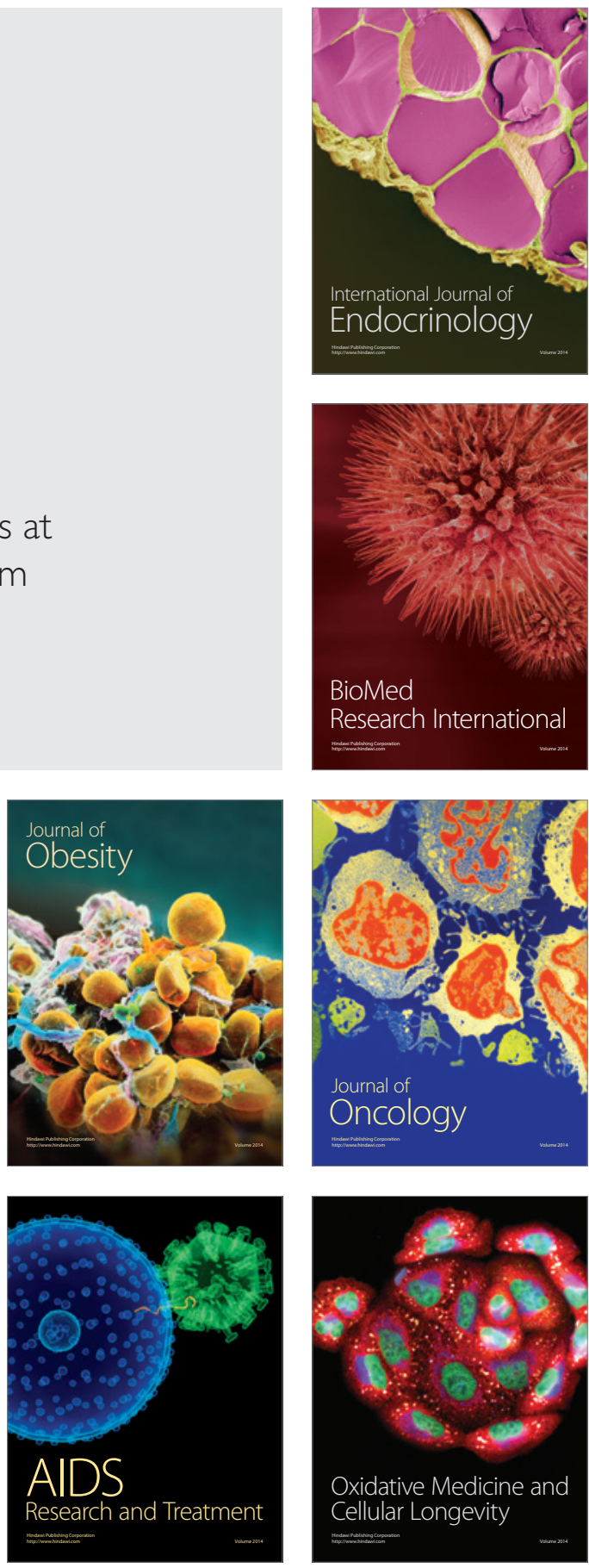